\title{
Spatial Patterns of Tuberculosis and Diabetes Mellitus in Los Angeles County, California
}

\section{Monica Rosales ( $\nabla$ mrosales@ph.lacounty.gov )}

LA County Department of Public Health https://orcid.org/0000-0003-0553-1540

\section{Edward Lan}

LA County Department of Public Health

\section{Ramon E. Guevara}

LA County Department of Public Health

Josephine L. Yumul

LA County Department of Public Health

Douglas M. Morales

LA County Department of Public Health

Julie M. Higashi

LA County Department of Public Health

Alicia H. Chang

LA County Department of Public Health

\section{Research article}

Keywords: public health, hot spots, racial/ethnic groups, Geographic Information Systems, health disparities

Posted Date: August 29th, 2019

DOI: https://doi.org/10.21203/rs.2.13623/v1

License: (c) (i) This work is licensed under a Creative Commons Attribution 4.0 International License.

Read Full License 


\section{Abstract}

Background Tuberculosis remains a public health problem that disproportionately affects vulnerable populations. The objective of the study was to apply Geographic Information Systems (GIS) methods to identify statistically significant hot spots of tuberculosis (TB) and diabetes mellitus (DM) and identify areas and populations that are disproportionately burdened by TB and DM.

Methods Verified cases of TB reported in Los Angeles County (LAC), California between 01/01/2015 and 06/30/2017 were identified from the LAC TB Control Program Surveillance Registry. The addresses for patients residing in LAC at the time of TB diagnosis were geocoded and mapped. Hot spot analyses were performed utilizing the Getis-Ord Gi* statistic to identify statistically significant hot spots of TB and DM.

Results Among 389 TB cases with DM, 43\% were Hispanic and 50\% were Asian. Geographic variations for Hispanic and Asian TB cases were found $(p<0.05)$. Hot spots of TB and DM were identified among Asians residing in the southwestern and southern regions of $L A C$ and among Hispanics residing in south central and northwestern LAC.

Conclusions GIS methods are important epidemiological tools for identifying and assessing geographic variations in disease morbidity. These findings highlight opportunities for public health interventions aimed at reducing health disparities in underserved communities.

\section{Background}

Despite a decline in active tuberculosis (TB) disease rates in recent decades, both globally ${ }^{1}$ and in the United States, ${ }^{2}$ TB remains a public health concern. TB affects many communities disproportionately, with disparities in TB incidence across racial/ethnic groups, socioeconomic status, and nativity. ${ }^{3}$ In Los Angeles County (LAC), California, ${ }^{4}$ the TB rate is higher than the rates in the state of California ${ }^{5}$ and in the United States, ${ }^{2}$ with the highest rates observed among Asian and Hispanic racial/ethnic groups.

The growing prevalence of diabetes mellitus (DM) is now a global health concern. ${ }^{6}$ In the past 3 decades, the global DM prevalence has increased from $4.7 \%$ to $8.5 \%$. The DM epidemic could contribute to an increase in TB burden and consequently pose a challenge to the World Health Organization's (WHO) "End TB Strategy" efforts ${ }^{7}$ to end TB. ${ }^{1,8}$ The co-occurrence of DM is a TB risk factor that can affect TB disease presentation and treatment response. ${ }^{9}$ Treatment of persons with DM poses a challenge for TB control programs since having DM increases the risk of progression from TB infection to active TB disease ${ }^{10}$ and contributes to poor outcomes. ${ }^{10-12}$ In LAC, $10 \%$ of residents have DM and $44 \%$ have prediabetes, ${ }^{13}$ similar to statewide estimates (9\% and $46 \%$, respectively). ${ }^{14}$ As the prevalence of DM increases, the number of people with TB and DM is also expected to rise. Furthermore, DM disproportionately affects racial/ethnic groups (e.g. Asian, Hispanic) in LAC and in California, ${ }^{14}$ groups that are also more likely to be faced with the co-occurrence of TB and DM. ${ }^{4}$ This is a concern in LAC, where in recent years $25 \%-30 \%$ of persons diagnosed with TB were reported to have DM. ${ }^{4}$ 
Targeted efforts to prevent TB in at-risk groups are urgently needed. However, initial steps must be taken to identify the areas and populations highly burdened by these diseases. The application of Geographic Information Systems (GIS) methods to public health research can elucidate the underlying geography of health disparities, which are not evident with traditional statistical analyses or statistical packages. GIS spatial analysis tools use geographic data to better understand risk factor-disease relationships ${ }^{15}$ and identify targets for public heath prevention and intervention. ${ }^{15,16} \mathrm{~A}$ better understanding of the epidemiology of TB and DM co-occurrence and the geographical context in which it occurs can aid our work toward achieving the goal of TB elimination. Thus, the objective of this study was to apply GIS methodology to identify geographic areas and populations that are disproportionately burdened by the co-occurrence of TB and DM in LAC, California.

\section{Methods}

We conducted a retrospective analysis in a cohort of verified cases of TB reported between 01/01/2015 and 06/30/2017 in LAC. TB cases were identified from the LAC TB Control Program Surveillance Registry. The TB Registry collects information on co-occurring medical conditions, including DM. This information is gathered through a variety of methods, including provider report, laboratory confirmation, and/or patient self-report. TB cases were grouped based on DM status (with DM or without DM). Descriptive statistics (e.g. frequencies, percentages) summarize the characteristics of TB cases. Demographic and clinical characteristics were compared between TB cases with DM and TB cases without DM. Chi-square tests were used to detect statistically significant $(p<0.05)$ differences in demographic and clinical characteristics among TB cases according to DM status. Descriptive statistics and chi-square test analyses were conducted in SAS Enterprise Guide 7.11.

Spatial analyses were conducted using ArcGIS 10.3.1. Addresses of incident TB cases residing in LAC at the time of TB diagnosis were successfully geocoded to obtain longitude and latitude coordinates and mapped using the cartographic base map of LAC, LAC 2010 census tracts map, and LAC health district boundaries map. Since LAC is comprised of 24 health districts that are used to plan and manage health service delivery, geocoded addresses were first spatially joined to their respective health district and summarized as choropleth maps to better assess distribution of TB and DM across LAC. Next, hot spot analyses using the Getis-Ord Gi* statistic $(p<0.05)$ were used to geographically assess the burden of TB and DM by census tracts. The Optimized Hot Spot Analysis tool in ArcMap 10.3 identifies statistically significant spatial clusters of high values (hot spots) and low values (cold spots). Areas with elevated burden of TB and DM were considered hot spots when confidence intervals ranged from $90 \%$ to $99 \%$. The map of LAC health district boundaries was overlaid on the LAC maps to determine which health districts were located within the hot spots.

The study was considered exempt by the LAC Department of Public Health Institutional Review Board. Informed consent was waived given that the data used were retrospectively collected and the work pertained to LAC TB Control Program's federal requirement to conduct active TB surveillance. 


\section{Results}

Among 1,346 TB cases, 389 (29\%) also had DM. Table 1 summarizes TB case characteristics by DM status. TB cases with DM were more likely to be older $(p<0.0001)$ and to be male $(p<0.01)$ than cases without DM. TB cases with DM were more likely to be Asian $(50 \%)$ or Hispanic $(43 \%),(p<0.01)$ than cases without DM. Compared to TB cases without DM, cases with DM were more likely to be born outside the U.S. (79\% vs. $91.2 \%$, respectively; $p<0.001)$. In terms of clinical characteristics, TB cases with DM were more likely to have pulmonary site of TB disease ( $80.7 \%$ vs. $69 \% ; p<0.0001)$, end stage renal disease $(9 \%$ vs. $2.7 \%$; $p<0.001)$, and more likely to have died with TB $(15.7 \%$ vs. $10.8 \% ; p<0.05)$, compared to TB cases without DM.

Since Hispanic and Asian TB cases accounted for $92 \%$ of TB cases with DM, only Asian and Hispanic cases were included in spatial analyses. Choropleth maps indicated elevated TB and DM burden among Asians residing in southwestern and southern LAC and among Hispanics residing in southcentral and northwestern LAC (results not shown). These findings were supported by hot spot analyses.

A statistically significant hot spot $(p<0.05)$ of elevated TB and DM burden was identified for all TB cases (Asian and Hispanic TB cases combined) in the southern central region of $L A C$, encompassing nine health districts (Figure 1). Statistically significant hot spots $(p<0.05)$ of elevated TB and DM burden were also identified by race/ethnicity. Among Asian TB cases, four hot spots were identified (Figure 2). These four hot spots were in the southwestern and southern regions of LAC, located in the following health districts: (1) Northeast, (2) Alhambra and East L.A., (3) El Monte and Pomona, and (4) Torrance and Harbor. Among Hispanic cases, two hot spots of elevated TB and DM were identified (Figure 3). These two hot spots were in the northwestern and southcentral regions of LAC and were in the following health districts: (1) Antelope Valley, and (2) Southwest, Southeast, East LA, Whittier, South, San Antonio, Inglewood and Compton.

\section{Discussion}

The co-occurrence of TB and DM has been recognized for centuries. ${ }^{9}$ In LAC, TB and DM disproportionately affect Asians and Hispanics. Furthermore, a majority (91\%) of TB cases with DM were born outside the U.S. Spatial analyses identified hot spots of TB and DM among Asian TB cases residing in the southwestern and southern regions of LAC. These regions correspond to areas in LAC with large Asian populations, ${ }^{14}$ with the southern region being an area where low English language proficiency is reported. ${ }^{14}$ TB and DM burden was identified among Hispanic TB cases residing in southcentral and northwestern LAC, regions with large concentrations of Hispanics with low English language proficiency. ${ }^{14}$

The elevated TB and DM burden delineated in the maps presented here are in line with reports and prior research indicating that TB and DM disproportionately and unequally affect racial/ethnic minorities and non-U.S. born persons. ${ }^{2,5,10,17,18}$ The co-occurrence of TB and DM should be closely examined by 
race/ethnicity given the increasing prevalence of $D M^{13}$ the growing non-U.S. born population in $L A C,{ }^{19}$ and the contribution of DM to poor TB treatment outcomes. ${ }^{10,17,20}$ Future research should focus on identifying areas where non-U.S. born individuals reside and examine the factors associated with disease co-occurrence. Also, spatial analyses by country of birth, primary language, and English language proficiency can inform when and where bilingual educational and culturally sensitive services are needed to reach target populations.

GIS methods serve as important epidemiological tools to identify geographic areas experiencing disproportionate burden of TB and DM and can help inform future activities and interventions. Interventions tailored to specific groups can aid in addressing TB morbidity and mortality and help continue our work towards reaching TB elimination. However, turning future activities and interventions into cost-effective and high-impact public health programs requires improvements in continuity of care delivery, through integration and coordination of care, across providers. For example, co-management of both TB and DM can be crucial to the optimal treatment and control of both diseases. Co-management would require collaboration from health professionals and patients to clearly assess all factors related to the monitoring and treatment of each disease. ${ }^{21} \mathrm{~A}$ disease co-management strategy would benefit from a patient education component that facilitates self-care by promoting adherence and completion of TB treatment and emphasizing the importance of disease monitoring and management. In fact, WHO's "Collaborative Framework for Care and Control of Tuberculosis and Diabetes" provides guidelines for integrated management of both diseases. ${ }^{6,22}$ Facilitating patient awareness and education for prevention, diagnosis, and treatment of TB and DM can help address health disparities among vulnerable populations.

While the strength of this paper is the application of GIS methodology to identify geographical areas and populations in LAC burdened by TB and DM, there are some limitations to these findings. Assessment of DM status was partly based on patient self-report. However, it should be noted that national estimates of DM incidence and prevalence are also based on self-report data. ${ }^{25}$ Furthermore, self-reported DM status is reliable and has high sensitivity and specificity. ${ }^{26,27,28}$ The data presented here are cross-sectional and do not indicate temporal order of onset of DM or TB. The TB and DM surveillance data do not provide information on severity or duration of DM among TB cases. Also, we did not examine the data by nativity to assess disease burden in areas where people from common nationalities reside. Despite these limitations, to our knowledge, this is one of the first set of analyses to examine spatial patterns of TB and DM burden.

\section{Conclusions}

In summary, our findings demonstrate the potential of GIS methods for identifying areas of elevated disease burden. Contextualizing disease burden geographically facilitates focusing and implementing community-based outreach initiatives, such as educational programs targeting both patients and providers, in areas at high risk for disease burden. These findings highlight opportunities for future public 
health interventions aimed at reducing health disparities in underserved communities. Geographical findings can guide prioritization of at-risk populations, identify those most likely to benefit from interventions, and can help monitor impact of prevention strategies and treatments.

\section{List Of Abbreviations}

DM: Diabetes Mellitus

GIS: Geographic Information Systems

LAC: Los Angeles County

TB: Tuberculosis

WHO: World Health Organization

\section{Declarations}

\section{Ethics approval and consent to participate}

The study was considered exempt by the LAC Department of Public Health Institutional Review Board. Informed consent was waived given that the data used were retrospectively collected and the work pertained to LAC TB Control Program's federal requirement to conduct active TB surveillance.

\section{Consent for publication}

Not applicable.

\section{Availability of data and material}

The datasets generated and/or analyzed for the current study are not publicly available in its entirety. However, reports that incorporate these data are available for download on the website (http://ph.lacounty.gov/tb/reports.htm). Also, a special data request can be submitted to request specific data presented here.

\section{Competing interests}

The authors declare that they have no competing interests.

\section{Funding}

No funding was allocated for this study.

\section{Authors' contributions}


All authors contributed to this article and have approved the final article. MR coordinated and led the development and design, data analyses, and writing of this article. MR, EL, JY analyzed the data. EL, JY, REG, DMM, JMH, AHC assisted with interpretation of the findings, editing, and write-up of this article.

\section{Acknowledgements}

Not applicable.

\section{References}

1. Global tuberculosis report 2018. Geneva: World Health Organization, 2018.

2. Reported Tuberculosis in the United States, 2017. Atlanta: Centers for Disease Control and Prevention, US Department of Health and Human Services, 2018.

3. Oren E. Health Disparities in Tuberculosis. In: Gerald L., Berry C, editors. Health Disparities in Respiratory Medicine: Humana Press, Cham; 2016. p. 207-35.

4. Tuberculosis in Los Angeles County: Surveillance Report 2016. Los Angeles: County of Los Angeles Department of Public Health Tuberculosis Control Program, 2017.

5. Report on Tuberculosis in California, 2016, 2017. Richmond, CA: California Department of Public Health Tuberculosis Control Branch, 2017.

6. Global report on diabetes. Geneva: World Health Organization, 2016.

7. The WHO End TB Strategy. Geneva: World Health Organization, 2015.

8. Al-Rifai RH PF, Critchley JA, Abu-Raddad LJ. Association between diabetes and active tuberculosis: A systematic review and meta-analyses. PLoS ONE. 2017;12(11):e0187967.

9. Dooley KE, Chaisson RE. Tuberculosis and diabetes mellitus: convergence of two epidemics. The Lancet Infectious diseases. 2009 Dec;9(12):737-46. PubMed PMID: 19926034. Pubmed Central PMCID: 2945809.

10. Demlow S, Oh P, Barry P. Increased risk of tuberculosis among foreign-born persons with diabetes in California, 2010-2012. BMC Public Health. 2015;15(263).

11. Baghaei P, Marjani M, Javanmard P, Tabarsi P, Masjedi M. Diabetes mellitus and tuberculosis facts and controversies. Journal of Diabetes \& Metabolic Disorders. 2013;12(1).

12. Sullivan T, Amor Y. The Co-Management of Tuberculosis and Diabetes: Challenges and Opportunities in the Developing World. PLoS Med. 2012;9(7).

13. Babey S, Wolstein J, Diamant A, Goldstein H. Prediabetes in California: Nearly Half of California Adults on Path to Diabetes. Los Angeles, CA: UCLA Center for Health Policy Research and California Center for Public Health Advocacy, 2016.

14. California Health Interview Survey (CHIS). Ask CHIS [Internet] [Accessed December 10, 2017]. Available from: http://ask.chis.ucla.edu 
15. Buehle C, Blevins M, Ossemane E, Gonzalez-Calvo L, Ndatimana E, Vermud S, et al. Assessing spatial patterns of HIV knowledge in rural Mozambique using geographic information systems. Tropical Medicine and International Health. 2015;20(3):353-64.

16. Agarwal A ND, Teeter LD, Graviss EA. Spatial-temporal distribution of genotyped tuberculosis cases in a county with active transmission. BMC Infectious Diseases. 2017;17(378).

17. Suwanpimolkul G, Grinsdale J, Jarlsberg, LG, Higashi J, Osmond D, Hopewell P, et al. Association between Diabetes Mellitus and Tuberculosis in United States-Born and Foreign-Born Populations in San Francisco. PLoS ONE. 2014;9(12):e114442.

18. Zhar, RS, Peterson, RA, Polgreen, LA, Cavanaugh, JE, Hornick, Winthrop, KL, et al. Diabetes as an increasingly common comorbidity among patient hospitalizations for tuberculosis in the USA. BMJ Open Diabetes Research and Care. 2016;4.

19. U.S. Census Bureau. State and County QuickFacts [Internet] [Accessed December 19, 2017]. Available from: https:www.census.gov/quickfacts/fact/table/losangelescountycalifornia/PS045216.

20. Baker M, Harries A, Jeon C, Hart J, Kapur A, Lonnroth K, et al. The impact of diabetes on tuberculosis treatment outcomes: A systematic review. BMC Medicine. 2011;9(81).

21. Makaroff LE, Hegelund MH. TB and Diabetes: Risks, management and improving outcomes. Diabetes Voice. 2016;61:26-9.

22. Collaborative framework for care and control of tuberculosis and diabetes. Geneva: World Health Organization \& International Union against Tuberculosis and Lung Disease, 2011.

23. Ali, MK, Siegel, KR, Laxy, M, Gregg, EW. Advancing Measurement of Diabetes at the Population Level. Current Diabetes Reports. 2018;18(108).

24. Vigen, C, Kwan, ML, John, EM, Gomez, SL, Kegan, THM, Lu, Y, et al. Validation of self-reported comorbidity status of breast cancer patients with meidcal records: the California Breast Cancer Survivorship Consortium (CBCSC). Cancer Causes Control. 2016;27(3).

25. Scheider, ALC, Pankow, JS, Heiss, G, Selvin, E. Validity and Reliability of self-reported Diabetes in the Atherosclerosis Risk in Communities Study. 2012;176(8): 738-743.

26. Jackson, JM, DeFor, TA, Crain AL, Kerby TJ, Strayer, LS, Lewis CE, et al. Validity of Diabetes SelfReports in the Women's Health Initiative. Menopause. 2014;21(8); 861-8.

\section{Tables}


Table 1. Characteristics of TB Cases by Diabetes Mellitus Status, Los Angeles County, California, 20152017

Diabetes Mellitus Status

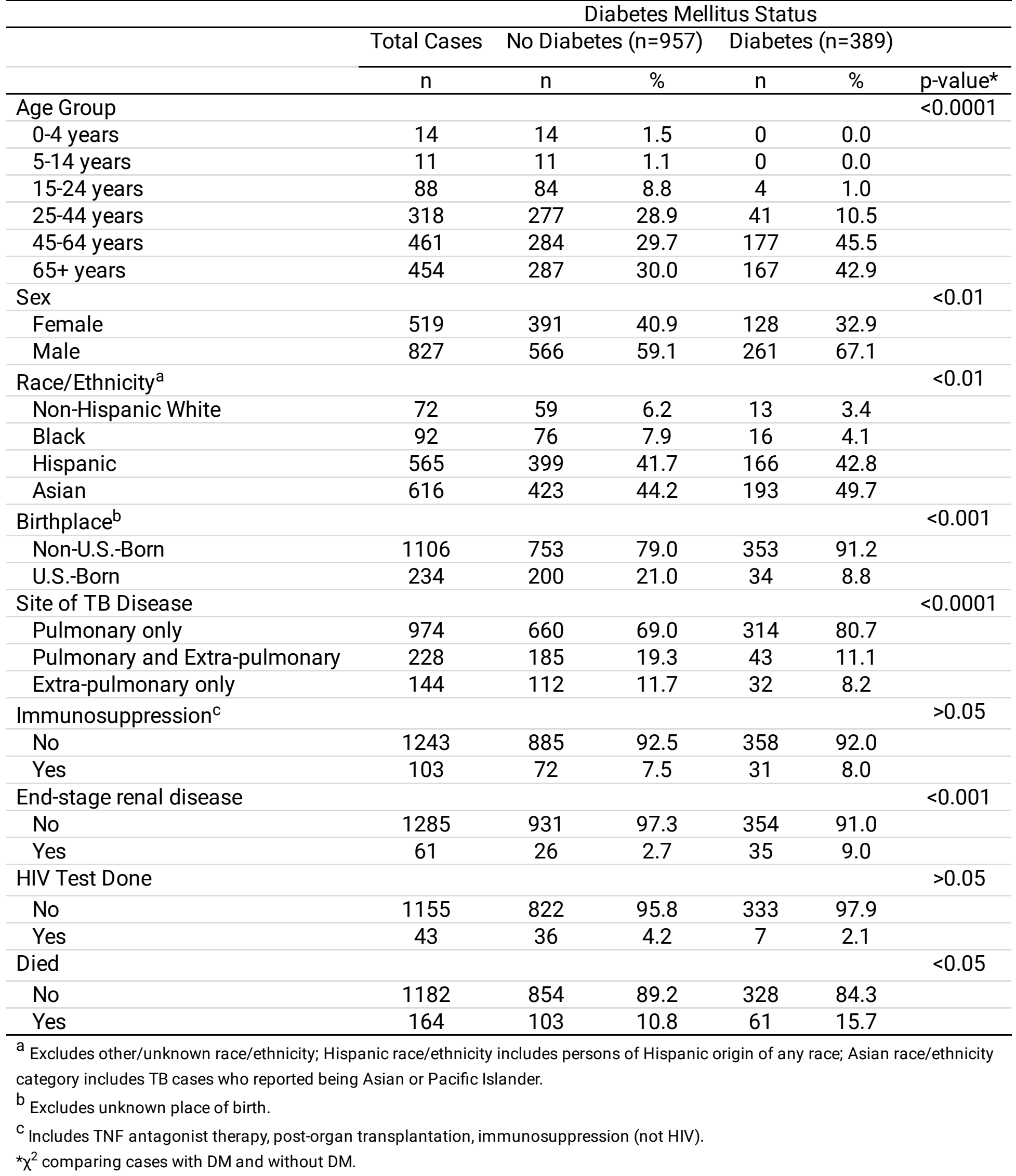

\section{Figures}




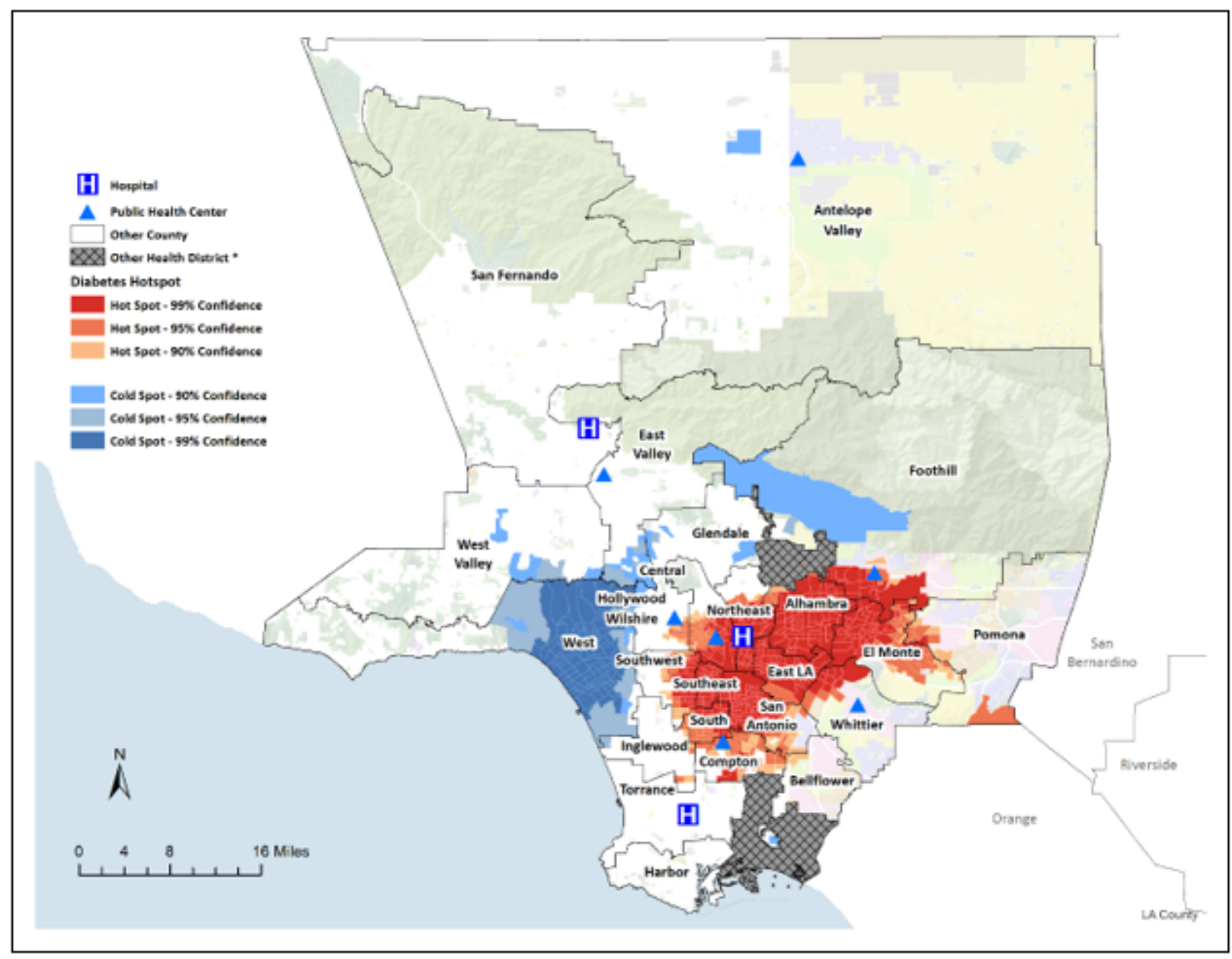

Figure 1

Co-occurrence of TB and DM in Los Angeles County, California, 2015-2017* *Optimized hot spot analysis of TB and DM burden in Los Angeles County, California, United States. Census tracts with elevated burden are represented by hot spots $(p<0.05)$; census tracts with lower burden are represented by cold spots $(p<0.05)$. The LAC health district map was overlaid on the hot spot map. Data exclude TB cases from Long Beach and Pasadena, as these cities have their own health departments. 


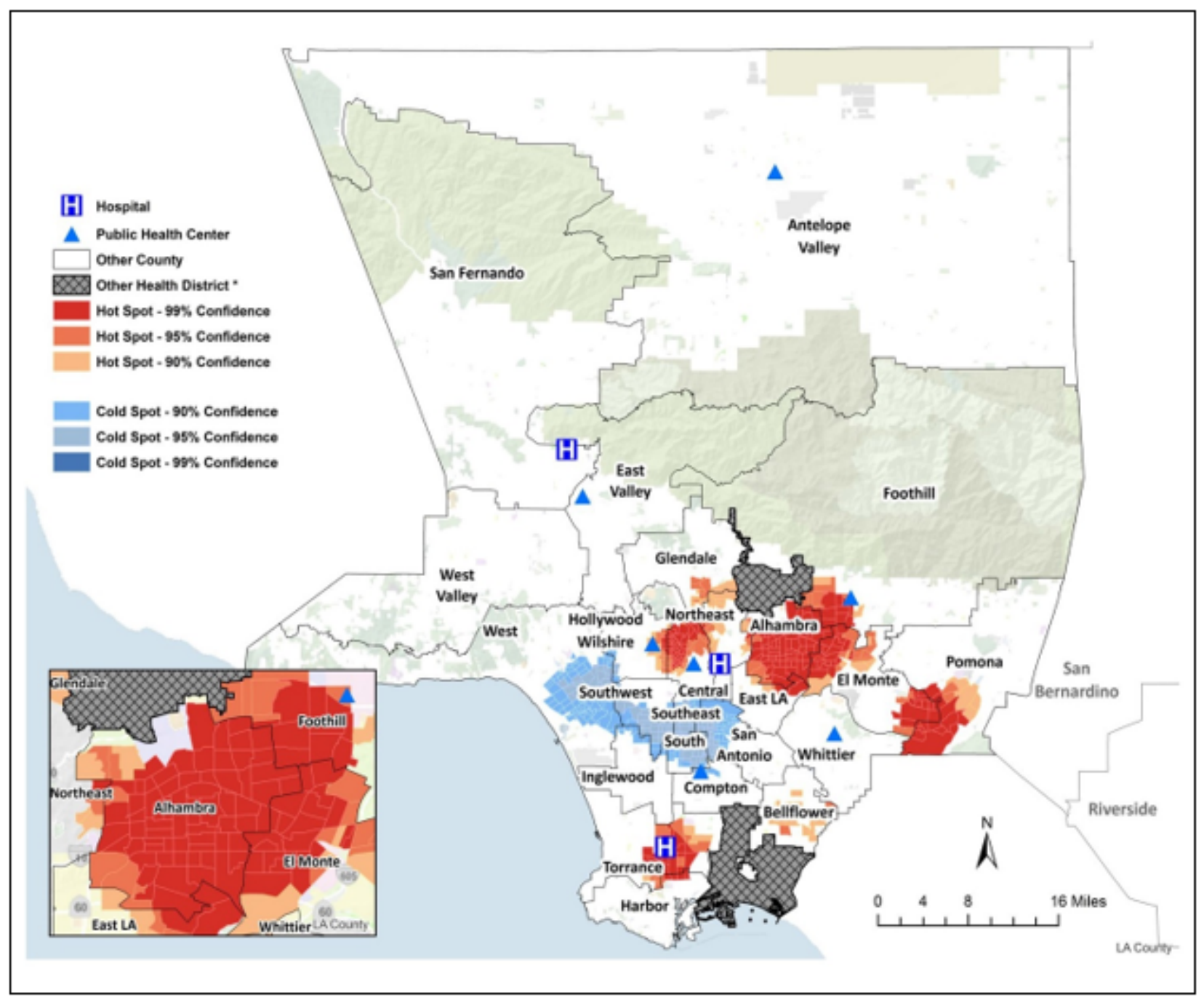

Figure 2

Co-Occurrence of TB and DM among Asians in Los Angeles County, California, 2015-2017* *Optimized hot spot analysis of TB and DM burden among Asian individuals in Los Angeles County, California, United States. Asian race/ethnicity category includes TB cases who reported being Asian or Pacific Islander. Census tracts with elevated burden are represented by hot spots $(p<0.05)$; census tracts with lower burden are represented by cold spots $(p<0.05)$. The LAC health district map was overlaid on the hot spot map. Data exclude TB cases from Long Beach and Pasadena, as these cities have their own health departments. 


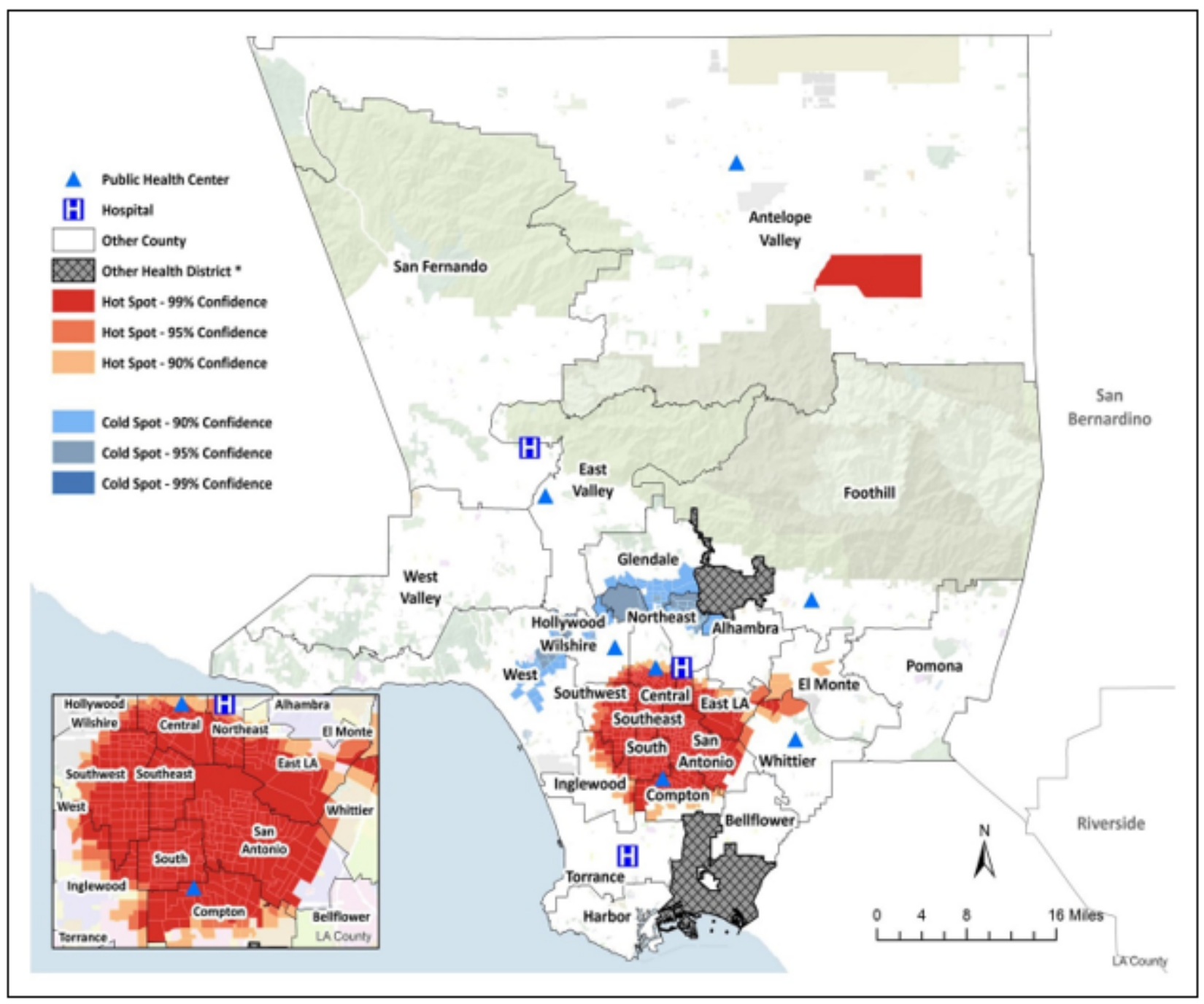

Figure 3

Co-Occurrence of TB and DM among Hispanics in Los Angeles County, California, 2015-2017* *Optimized hot spot analysis of TB and DM burden among Hispanic individuals in Los Angeles County, California, United States. Hispanic race/ethnicity includes persons of Hispanic origin of any race. Census tracts with elevated burden are represented by hot spots $(\mathrm{p}<0.05)$; census tracts with lower burden are represented by cold spots $(p<0.05)$. The LAC health district map was overlaid on the hot spot map. Data exclude TB cases from Long Beach and Pasadena, as these cities have their own health departments. 